\title{
Approximation Properties and $q$-Statistical Convergence of Stancu-Type Generalized Baskakov-Szász Operators
}

\author{
Qing-Bo Cai $\mathbb{D},{ }^{1}$ Adem Kilicman $\mathbb{D}^{2},{ }^{2}$ and Mohammad Ayman Mursaleen $\mathbb{D}^{2,3}$ \\ ${ }^{1}$ Fujian Provincial Key Laboratory of Data-Intensive Computing, Key Laboratory of Intelligent Computing and \\ Information Processing, School of Mathematics and Computer Science, Quanzhou Normal University, Quanzhou 362000, China \\ ${ }^{2}$ Department of Mathematics and Statistics, Faculty of Science, Universiti Putra Malaysia, 43400 Serdang, Selangor, Malaysia \\ ${ }^{3}$ School of Information and Physical Sciences, The University of Newcastle, Callaghan, NSW 2308, Australia
}

Correspondence should be addressed to Mohammad Ayman Mursaleen; mohdaymanm@gmail.com

Received 31 October 2021; Accepted 18 December 2021; Published 20 January 2022

Academic Editor: Mikail Et

Copyright (c) 2022 Qing-Bo Cai et al. This is an open access article distributed under the Creative Commons Attribution License, which permits unrestricted use, distribution, and reproduction in any medium, provided the original work is properly cited.

In this article, we introduce Stancu-type modification of generalized Baskakov-Szász operators. We obtain recurrence relations to calculate moments for these new operators. We study several approximation properties and $q$-statistical approximation for these operators.

\section{Introduction}

In 1912, Bernstein [1] proposed the famous polynomial known as the Bernstein polynomial to give a simple, short, and most elegant proof of the Weierstrass approximation theorem. Since then, several papers have appeared to study approximation properties in different settings and spaces. Many new operators were constructed, e.g., Szász [2], Mirakjan [3], Kantorovic [4], Durrmeyer [5], Stancu [6], and many more [7-9]. These operators provide the improvement of approximating functions of different classes and give better and better estimates. For example, the Baskakov operators were given in [10]:

$$
V_{p}(\mathfrak{h} ; \mathfrak{v})==\sum_{l=0}^{\infty}\left(\begin{array}{c}
p+l-1 \\
l
\end{array}\right) \frac{\mathfrak{v}^{l}}{(1+\mathfrak{b})^{p+l}} \mathfrak{h}\left(\frac{l}{p}\right)
$$

For $\mathfrak{h} \in C[0, \infty)$, the space of all continuous functions on $[0$, $\infty)$ normed with standard sup-norm $\|\cdot\|_{\infty}$.

Devore and Lorentz [11] introduced a generalization of operators (1) dependent on a constant $a>0$ and independent of $p$ as follows:

$$
B_{p}(\mathfrak{h} ; u)=\sum_{j=0}^{\infty} W_{p, j}^{a}(u) \mathfrak{h}\left(\frac{j}{p}\right)
$$

where

$$
\begin{aligned}
W_{p, j}^{a}(u) & =e^{-a u /(1+u)} \frac{G_{j}(p, a)}{j !} \frac{u^{j}}{(1+u)^{p+j}}, \sum_{j=0}^{\infty} W_{p, j}^{a}(u)=1, \\
G_{j}(p, a) & =\sum_{i=0}^{j}\left(\begin{array}{l}
j \\
i
\end{array}\right)(p)_{i} a^{j-i},
\end{aligned}
$$

and $(p)_{i}=p(p+1) \cdots(p+i-1),(p)_{0}=1$.

Recently, Agrawal et al. [12] studied the following operators (2):

$$
L_{p, a}^{*}(\mathfrak{h} ; u)=p \sum_{j=0}^{\infty} W_{p, j}^{a}(u) \int_{0}^{\infty} s_{p, j}(t) \mathfrak{h}(t) d t,
$$

for $\mathfrak{h} \in C_{\gamma}[0, \infty):=\left\{\mathfrak{h} \in C[0, \infty):|\mathfrak{h}(t)| \leq M_{\mathfrak{h}} e^{\gamma t}\right.$, for some $\gamma$ $\left.>0, M_{\mathfrak{h}}>0\right\}$, where $s_{p, j}(t)=e^{-p t}\left((p t)^{j} / j !\right)$.

Inspired by Stancu's work [6], we have studied recently the Stancu-type generalization in [13]. Now, we propose the Stancu-type generalization of operators (4) as follows: 


$$
\mathbf{\Omega}_{p, a}^{(\lambda, \mu)}(\mathfrak{h} ; \mathfrak{v})=p \sum_{k=0}^{\infty} W_{p, k}^{a}(\mathfrak{v}) \int_{0}^{\infty} s_{p, k}(t) \mathfrak{h}\left(\frac{p t+\lambda}{p+\mu}\right) d t
$$

for any bounded and integrable function $\mathfrak{h}$ defined on $[0, \infty)$ , where $0 \leq \lambda \leq \mu$. For $\lambda=\mu=0$, the operators (5) reduce to operators (4).

We establish recurrence relations to find moments and central moments. We study some approximation properties and the Voronovskaja-type asymptotic formula. We also study weighted approximation.

\section{Auxiliary Results}

Our first result is the recurrence formula for moments.

Theorem 1. The $m^{\text {th }}$ order moment for (5) is defined by

$$
\begin{aligned}
\mathfrak{T}_{p, a, m}^{(\lambda, \mu)}(\mathfrak{b}):= & \mathbf{Q}_{p, a}^{(\lambda, \mu)}\left(t^{m} ; \mathfrak{v}\right)=p \sum_{k=0}^{\infty} W_{p, k}^{a}(\mathfrak{b}) \int_{0}^{\infty} s_{p, k}(t) \mathfrak{h} \\
& \cdot\left(\frac{p t+\lambda}{p+\mu}\right)^{m} d t .
\end{aligned}
$$

$$
m=0,1,2, \cdots . \text { Then, } \mathfrak{\mathfrak { T }}_{p, a, 0}^{(\lambda, \mu)}(\mathfrak{v})=1, \text { and }
$$

$$
\begin{aligned}
(m+1)(1+\mathfrak{v}) \mathfrak{I}_{p, a, m+1}^{(\lambda, \mu)}(\mathfrak{v}) & \\
= & \mathfrak{v}(1+\mathfrak{v})^{2}\left[\mathfrak{I}_{p, a, m}^{(\lambda, \mu)}(\mathfrak{v})\right]^{\prime}+\{(1+\mathfrak{v})(\lambda+p \mathfrak{v}+m \\
& +1)+a \mathfrak{v}\} \mathfrak{\mathfrak { I }}_{p, a, m}^{(\lambda, \mu)}(\mathfrak{v})-\frac{\lambda m}{p+\mu}(1+\mathfrak{v}) \mathfrak{T}_{p, a, m-1}^{(\lambda, \mu)}(\mathfrak{b})
\end{aligned}
$$

Proof. We use the identity

$$
\mathfrak{v}(1+\mathfrak{v})^{2}\left(W_{p, k}^{a}(\mathfrak{b})\right)^{\prime}=[(k-p \mathfrak{v})(1+\mathfrak{v})-a \mathfrak{v}] W_{p, k}^{a}(\mathfrak{v}) .
$$

Then,

$$
\begin{aligned}
\mathfrak{v}(1+\mathfrak{v})^{2}\left[\mathfrak{I}_{p, a, m}^{(\lambda, \mu)}(\mathfrak{v})\right]^{\prime} \\
=p \sum_{k=0}^{\infty} \mathfrak{v}(1+\mathfrak{v})^{2}\left(W_{p, k}^{a}(\mathfrak{v})\right)^{\prime} \int_{0}^{\infty} s_{p, k}(t) \mathfrak{h}\left(\frac{p t+\lambda}{p+\mu}\right)^{m} d t \\
=p \sum_{k=0}^{\infty}[(k-p \mathfrak{v})(1+\mathfrak{v})-a \mathfrak{v}] W_{p, k}^{a}(\mathfrak{b}) \int_{0}^{\infty} s_{p, k}(t)\left(\frac{p t+\lambda}{p+\mu}\right)^{m} d t \\
=p(1+\mathfrak{v}) \sum_{k=0}^{\infty}(k-p \mathfrak{v}) W_{p, k}^{a}(\mathfrak{b}) \int_{0}^{\infty} s_{p, k}(t)\left(\frac{p t+\lambda}{p+\mu}\right)^{m} d t \\
\quad-a \mathfrak{v} \cdot p \sum_{k=0}^{\infty} W_{p, k}^{a}(\mathfrak{v}) \int_{0}^{\infty} s_{p, k}(t)\left(\frac{p t+\lambda}{p+\mu}\right)^{m} d t,
\end{aligned}
$$

$$
\mathfrak{v}(1+\mathfrak{v})^{2}\left[\mathfrak{\mathfrak { T }}_{p, a, m}^{(\lambda, \mu)}(\mathfrak{v})\right]^{\prime}=I-a x \mathfrak{T}_{p, a, m}^{(\lambda, \mu)}(\mathfrak{v}),
$$

where

$$
\begin{aligned}
I= & p(1+\mathfrak{v}) \sum_{k=0}^{\infty}(k-p \mathfrak{v}) W_{p, k}^{a}(\mathfrak{v}) \int_{0}^{\infty} s_{p, k}(t)\left(\frac{p t+\lambda}{p+\mu}\right)^{m} d t \\
= & p(1+\mathfrak{v}) \sum_{k=0}^{\infty}[k-(p t+\lambda)+(p t+\lambda)-p \mathfrak{v}] \\
& \cdot W_{p, k}^{a}(\mathfrak{v}) \int_{0}^{\infty} s_{p, k}(t)\left(\frac{p t+\lambda}{p+\mu}\right)^{m} d t \\
= & p(1+\mathfrak{v}) \sum_{k=0}^{\infty}[(k-p t)+(p t+\lambda)-(\lambda+p \mathfrak{v})] \\
& \cdot W_{p, k}^{a}(\mathfrak{v}) \int_{0}^{\infty} s_{p, k}(t)\left(\frac{p t+\lambda}{p+\mu}\right)^{m} d t \\
= & p(1+\mathfrak{v}) \sum_{k=0}^{\infty} W_{p, k}^{a}(\mathfrak{b}) \int_{0}^{\infty}(k-p t) s_{p, k}(t)\left(\frac{p t+\lambda}{p+\mu}\right)^{m} d t \\
& +p(1+\mathfrak{v})(p+\mu) \sum_{k=0}^{\infty} W_{p, k}^{a}(\mathfrak{v}) \int_{0}^{\infty}(k-p t) s_{p, k}(t)\left(\frac{p t+\lambda}{p+\mu}\right)^{m+1} \\
& \cdot d t-p(1+\mathfrak{v})(\lambda+p \mathfrak{v}) \sum_{k=0}^{\infty} W_{p, k}^{a}(\mathfrak{v}) \int_{0}^{\infty} s_{p, k}(t)\left(\frac{p t+\lambda}{p+\mu}\right)^{m} d t
\end{aligned}
$$

$$
I=\sum_{1}+\sum_{2}+\sum_{3}, \text { say }
$$

where

$$
\sum_{2}=(1+\mathfrak{v})(p+\mu) \mathfrak{T}_{p, a, m+1}^{(\lambda, \mu)}(\mathfrak{b})
$$

$$
\sum_{3}=-(1+\mathfrak{b})(\lambda+p \mathfrak{v}) \mathfrak{T}_{p, a, m}^{(\lambda, \mu)}(\mathfrak{v})
$$

$$
\begin{aligned}
\sum_{1}= & p(1+\mathfrak{v}) \sum_{k=0}^{\infty} W_{p, k}^{a}(\mathfrak{v}) \int_{0}^{\infty}(k-p t) s_{n, k}(t)\left(\frac{p t+\lambda}{p+\mu}\right)^{m} d t \\
= & (1+\mathfrak{v}) \sum_{k=0}^{\infty} W_{p, k}^{a}(\mathfrak{v}) \int_{0}^{\infty} p t\left(s_{p, k}(t)\right)^{\prime} \\
& \cdot\left(\frac{p t+\lambda}{p+\mu}\right)^{m} d t,\left(\operatorname{using} t\left(s_{p, k}(t)\right)^{\prime}=\int_{0}^{\infty}(k-p t) s_{p, k}(t)\right), \\
= & (p+\mu)(1+\mathfrak{b}) \sum_{k=0}^{\infty} W_{p, k}^{a}(\mathfrak{v}) \int_{0}^{\infty}\left(\frac{p t+\lambda}{p+\mu}-\frac{\lambda}{p+\mu}\right) \\
& \cdot\left(s_{p, k}(t)\right)^{\prime}\left(\frac{p t+\lambda}{p+\mu}\right)^{m} d t=(p+\mu)(1+\mathfrak{v}) \\
& \cdot \sum_{k=0}^{\infty} W_{p, k}^{a}(\mathfrak{v}) \int_{0}^{\infty}\left(s_{p, k}(t)\right)^{\prime}\left(\frac{p t+\lambda}{p+\mu}\right)^{m+1} d t \\
& -\lambda(1+\mathfrak{v}) \sum_{k=0}^{\infty} W_{p, k}^{a}(\mathfrak{v}) \int_{0}^{\infty}\left(s_{p, k}(t)\right)^{\prime}\left(\frac{p t+\lambda}{p+\mu}\right)^{m} d t \\
= & \mathscr{J}_{1}+\mathscr{J}_{2}, \text { say }
\end{aligned}
$$


where

$$
\begin{aligned}
\mathscr{J}_{1}= & (p+\mu)(1+\mathfrak{v}) W_{p, k}^{a}(\mathfrak{v}) \int_{0}^{\infty}\left(s_{p, k}(t)\right)^{\prime}\left(\frac{p t+\lambda}{p+\mu}\right)^{m+1} d t \\
= & -(1+\mathfrak{v})(m+1) p \sum_{k=0}^{\infty} W_{p, k}^{a}(\mathfrak{v}) \int_{0}^{\infty} s_{p, k}(t)\left(\frac{p t+\lambda}{p+\mu}\right)^{m} d t \\
& -(1+\mathfrak{v})(m+1) \mathfrak{T}_{p, a, m}^{(\lambda, \mu)}(\mathfrak{v}), \\
\mathscr{J}_{2}= & -\lambda(1+\mathfrak{v}) \sum_{k=0}^{\infty} W_{p, k}^{a}(\mathfrak{v}) \int_{0}^{\infty}\left(s_{p, k}(t)\right)^{\prime}\left(\frac{p t+\lambda}{p+\mu}\right)^{m} d t \\
= & -\lambda(1+\mathfrak{v})\left(\frac{-m n}{p+\mu}\right) \sum_{k=0}^{\infty} W_{p, k}^{a}(\mathfrak{v}) \int_{0}^{\infty} s_{p, k}(t)\left(\frac{p t+\lambda}{p+\mu}\right)^{m-1} d t \\
= & \left(\frac{-m \lambda}{p+\mu}\right)(1+\mathfrak{v}) \mathfrak{T}_{p, a, m-1}^{(\lambda, \mu)}(\mathfrak{v}) .
\end{aligned}
$$

Therefore,

$$
\sum_{1}=\left(\frac{-m \lambda}{p+\mu}\right)(1+\mathfrak{v}) \mathfrak{T}_{p, a, m-1}^{(\lambda, \mu)}(\mathfrak{v})-(1+\mathfrak{b})(m+1) \mathfrak{T}_{p, a, m}^{(\lambda, \mu)}(\mathfrak{b}) .
$$

Substituting (17), (14), and (13) in (12), we get

$$
\begin{aligned}
I= & \frac{-m \lambda}{p+\mu}(1+\mathfrak{v}) \mathfrak{I}_{p, a, m-1}^{(\lambda, \mu)}(\mathfrak{b})-(1+\mathfrak{v})(p \mathfrak{v}+\lambda \\
& +m+1) \mathfrak{I}_{p, a, m}^{(\lambda, \mu)}(\mathfrak{b})+(1+\mathfrak{b})(p+\mu) \mathfrak{I}_{p, a, m+1}^{(\lambda, \mu)}(\mathfrak{b}) .
\end{aligned}
$$

Further, substituting (18) in (10), we get the result.

Corollary 2. From the above theorem, we get

(i) $\mathfrak{Q}_{p, a}^{(\lambda, \mu)}(1 ; \mathfrak{v})=1$

(ii) $\mathfrak{\Omega}_{p, a}^{(\lambda, \mu)}(t ; \mathfrak{v})=1 /(p+\mu)(p \mathfrak{v}+(a \mathfrak{v} /(1+\mathfrak{v}))+1+\lambda)$

(iii) $\mathbf{\Omega}_{p, a}^{(\lambda, \mu)}\left(t^{2} ; \mathfrak{b}\right)=1 /(p+\mu)^{2}\left[\left\{p^{2}+p+\left(a^{2} /(1+\mathfrak{v})^{2}\right)+\right.\right.$ $(2 a p /(1+\mathfrak{b}))\} \mathfrak{v}^{2}+\{4 p+(4 a /(1+\mathfrak{v}))+2 \lambda p+(2 a \lambda /$ $\left.(1+\mathfrak{v}))\} \mathfrak{v}+\left\{\lambda^{2}+2 \lambda+2\right\}\right]$

Theorem 3. The $m^{\text {th }}$ order central moment is defined by

$$
\begin{aligned}
\mathfrak{M}_{p, a, m}^{(\lambda, \mu)}(\mathfrak{v}) & :=\mathfrak{Q}_{p, a}^{(\lambda, \mu)}\left((t-\mathfrak{v})^{m} ; \mathfrak{v}\right) \\
& =p \sum_{k=0}^{\infty} W_{p, k}^{a}(\mathfrak{v}) \int_{0}^{\infty} s_{p, k}(t) \mathfrak{h}\left(\frac{p t+\lambda}{p+\mu}-u\right)^{m} d t .
\end{aligned}
$$

$m=0,1,2, \cdots$. The following recurrence relation holds:

$$
\begin{aligned}
\mathfrak{v}(1+ & \mathfrak{v})^{2}\left(\mathfrak{M}_{p, a, m+1}^{(\lambda, \mu)}(\mathfrak{v})\right)^{\prime} \\
= & \left(\frac{1+\mathfrak{v}}{p+\mu}\right)\left(\lambda-p \mathfrak{v}-\mu \mathfrak{v}-m p \mathfrak{v}-m \mu \mathfrak{v}-m p \mathfrak{v}^{2}\right. \\
& \left.-m \mu \mathfrak{v}^{2}\right) \mathfrak{M}_{p, a, m-1}^{(\lambda, \mu)}(\mathfrak{v})-(m+m \mathfrak{v}+1+\mathfrak{v} \\
& \left.-\mu \mathfrak{v}+\lambda-\mu \mathfrak{v}^{2}+\lambda \mathfrak{v}+a \mathfrak{v}\right) \mathfrak{M}_{p, a, m}^{(\lambda, \mu)}(\mathfrak{v}) \\
& +(1+\mathfrak{v})(p+\mu) \mathfrak{M}_{p, a, m+1}^{(\lambda, \mu)}(\mathfrak{v}) .
\end{aligned}
$$

Corollary 4. From the above theorem, we get

(a) $\boldsymbol{\Omega}_{p, a}^{(\lambda, \mu)}((t-\mathfrak{v}) ; \mathfrak{v})=1 /(p+\mu)(-\mu \mathfrak{v}+(a \mathfrak{v} /(1+\mathfrak{v}))+\lambda$ $+1)$

(b) $\mathfrak{Q}_{p, a}^{(\lambda, \mu)}\left((t-\mathfrak{v})^{2} ; \mathfrak{v}\right)=1 /(p+\mu)^{2}\left(p+\mu^{2}+\left(a^{2} /(1+\mathfrak{v})^{2}\right)\right.$ $-(2 a \mu /(1+\mathfrak{b}))) \mathfrak{b}^{2}+\left(2 /(p+\mu)^{2}\right)(p-\mu-\lambda \mu+((2+$ $\lambda) a /(1+\mathfrak{v}))) \mathfrak{v}+\left(1 /(p+\mu)^{2}\right)\left(\lambda^{2}+2 \lambda+2\right)$

Corollary 5. We further get
(a) $\lim _{p \longrightarrow \infty} p \mathfrak{Q}_{p, a}^{(\lambda, \mu)}((t-\mathfrak{b}) ; \mathfrak{b})=-\mu u+(a \mathfrak{v} / 1+\mathfrak{v})+\lambda+$ 1
(b) $\lim _{p \longrightarrow \infty} p \mathfrak{Q}_{p, a}^{(\lambda, \mu)}\left((t-\mathfrak{v})^{2} ; \mathfrak{v}\right)=\mathfrak{v}(\mathfrak{v}+2)$

\section{Main Results}

Peetre's $K$-functional is defined as

$$
K_{2}(\mathfrak{h}, \delta):=\inf \left\{\|\mathfrak{h}-\mathfrak{g}\|+\delta\left\|\mathfrak{g}^{\prime \prime}\right\|: \mathfrak{g} \in C_{B}^{2}[0, \infty)\right\}
$$

for $\mathfrak{h} \in C_{B}[0, \infty), \delta>0$, where $C_{B}[0, \infty):=\left\{\mathfrak{h} \in C_{B}[0, \infty): \mathfrak{h}\right.$ is bounded on $[0, \infty)\}$ and $C_{B}^{2}[0, \infty):=\left\{\mathfrak{g} \in C_{B}[0, \infty): \mathfrak{g}^{\prime}, \mathfrak{g}^{\prime \prime}\right.$ $\left.\in C_{B}[0, \infty)\right\}$. Note that

$$
K_{2}(\mathfrak{h} ; \delta) \leq M \omega_{2}(\mathfrak{h} ; \sqrt{\delta}), M>0,
$$

where $\omega_{2}(\mathfrak{h} ; \delta)$ is the second-order modulus of continuity [11].

$$
\omega_{2}(\mathfrak{h}, \delta)=\sup _{0<l \leq \delta \mathfrak{b} \in 0, \infty)} \sup _{0}|\mathfrak{h}(\mathfrak{b}+2 l)-2 \mathfrak{h}(\mathfrak{b}+l)+\mathfrak{h}(\mathfrak{b})|, \quad \delta>0 .
$$

The usual modulus of continuity of $\mathfrak{h} \in C_{B}[0, \infty)$ is defined as

$$
\omega_{1}(\mathfrak{h}, \delta)=\sup _{0<l \leq \delta \mathfrak{v} \in 0, \infty)} \sup _{\mid}|\mathfrak{h}(\mathfrak{v}+l)-\mathfrak{h}(\mathfrak{v})|
$$


Theorem 6. For $\mathfrak{h} \in C_{B}[0, \infty)$,

$$
\begin{aligned}
\left|\mathfrak{Q}_{p, a}^{(\lambda, \mu)}(\mathfrak{h} ; \mathfrak{v})-\mathfrak{h}(\mathfrak{v})\right| \leq & M \omega_{2}\left(\mathfrak{h} ; \sqrt{\phi_{p, a}^{(\lambda, \mu)}}\right)+\omega_{1}\left(\mathfrak{h} ; \frac{1}{p+\mu}\right. \\
& \left.\cdot\left(1+\lambda-\mu \mathfrak{v}+\frac{a \mathfrak{v}}{1+\mathfrak{v}}\right)\right)
\end{aligned}
$$

where $M>0$ and

$$
\begin{aligned}
\phi_{p, a}^{(\lambda, \mu)}= & \frac{1}{(p+\mu)^{2}}\left\{\left(p^{2}+\mu^{2}+\frac{a^{2}}{(1+\mathfrak{v})^{2}}-\frac{2 a \mu}{(1+\mathfrak{v})}\right) \mathfrak{v}^{2}\right. \\
& \left.+\left(2 p-2 \mu-2 \lambda \mu+\frac{2 a(2+\lambda)}{(1+\mathfrak{v})}\right) \mathfrak{v}\right\} \\
& +\frac{1}{(p+\mu)^{2}}\left\{\left(1+\lambda-\mu \mathfrak{v}+\frac{a \mathfrak{v}}{1+\mathfrak{v}}\right)^{2}+\lambda^{2}+2 \lambda+2\right\} .
\end{aligned}
$$

Proof. Put

$$
\begin{aligned}
\tilde{\mathfrak{Q}}_{p, a}^{(\lambda, \mu)}(\mathfrak{h} ; \mathfrak{v})= & \mathfrak{Q}_{p, a}^{(\lambda, \mu)}(\mathfrak{h} ; \mathfrak{v})+\mathfrak{h}(\mathfrak{v})-\mathfrak{h}\left(\frac{1+\lambda}{p+\mu}+\frac{p \mathfrak{v}}{p+\mu}\right. \\
& \left.+\frac{1}{p+\mu} \frac{a \mathfrak{v}}{1+\mathfrak{b}}\right) .
\end{aligned}
$$

Note that $\tilde{\mathfrak{Q}}_{p, a}^{(\lambda, \mu)}(1 ; \mathfrak{v})=1$ and $\tilde{\mathbf{Q}}_{p, a}^{(\lambda, \mu)}(t ; \mathfrak{v})=\mathfrak{v}$. Let $\mathfrak{\mathfrak { E }} \in$ $C_{B}^{2}[0, \infty)$. Then, by using Taylor's theorem, we may write

$$
\mathfrak{F}(t)=\mathfrak{F}(\mathfrak{v})+(t-\mathfrak{v}) \mathfrak{F}^{\prime}(x) \int_{\mathfrak{v}}^{t}(t-y) \mathfrak{F}^{\prime \prime}(y) d y,
$$

which gives

$$
\begin{aligned}
& \tilde{\mathbf{\Omega}}_{p, a}^{(\lambda, \mu)}(\mathfrak{F} ; \mathfrak{b})-\mathfrak{E}(\mathfrak{b}) \\
& =\mathfrak{F}^{\prime}(\mathfrak{v}) \tilde{\mathbf{Q}}_{p, a}^{(\lambda, \mu)}((t-\mathfrak{b}) ; \mathfrak{b})+\tilde{\mathbf{Q}}_{p, a}^{(\lambda, \mu)} \\
& \cdot\left(\int_{\mathfrak{v}}^{t}(t-y) \mathfrak{F}^{\prime \prime}(y) d y ; \mathfrak{v}\right) \\
& =\tilde{\mathbf{Q}}_{p, a}^{(\lambda, \mu)}\left(\int_{\mathfrak{v}}^{t}(t-y) \mathfrak{E}^{\prime \prime}(y) d y ; \mathfrak{v}\right) \\
& =\mathfrak{Q}_{p, a}^{(\lambda, \mu)}\left(\int_{\mathfrak{v}}^{t}(t-y) \mathfrak{E}^{\prime \prime}(y) d y ; \mathfrak{v}\right) \\
& -\int_{\mathfrak{v}}^{1 /(p+\mu)(1+\lambda+p \mathfrak{v}+(a \mathfrak{b} / 1+\mathfrak{b}))}\left(\frac{1+\lambda}{p+\mu}\right. \\
& \left.+\frac{p \mathfrak{v}}{p+\mu}+\frac{1}{p+\mu} \frac{a \mathfrak{v}}{1+\mathfrak{v}}-\mathfrak{v}\right) \mathfrak{F}^{\prime \prime}(y) d y .
\end{aligned}
$$

Hence,

$$
\begin{aligned}
\left|\tilde{\mathfrak{Q}}_{p, a}^{(\lambda, \mu)}(\mathfrak{F} ; \mathfrak{v})-\mathfrak{E}(\mathfrak{v})\right| \\
\leq \mathbf{\Omega}_{p, a}^{(\lambda, \mu)}\left(\left|\int_{\mathfrak{v}}^{t}(t-y) \mathfrak{F}^{\prime \prime}(y) d y\right| ; \mathfrak{v}\right) \\
\quad+\mid \int_{\mathfrak{v}}^{1 /(p+\mu)(1+\lambda+p \mathfrak{v}+(a \mathfrak{v} /(1+\mathfrak{v})))}\left(\frac{1+\lambda}{p+\mu}+\frac{p \mathfrak{v}}{p+\mu}\right. \\
\left.\quad+\frac{1}{p+\mu} \frac{a \mathfrak{v}}{1+\mathfrak{b}}-y\right) \mathfrak{E}^{\prime \prime}(y) d y \mid .
\end{aligned}
$$

Since $\left|\int_{\mathfrak{b}}^{t}(t-y) \mathfrak{E}^{\prime \prime}(y) d y\right| \leq(t-\mathfrak{v})^{2}\left\|\mathfrak{E}^{\prime \prime}\right\|$ and

$$
\begin{aligned}
& \mid \int_{\mathfrak{v}}^{1 /(p+\mu)(1+\lambda+p \mathfrak{v}+(a \mathfrak{b} /(1+\mathfrak{b})))}\left(\frac{1+\lambda}{p+\mu}+\frac{p \mathfrak{v}}{p+\mu}\right. \\
& \left.\quad+\frac{1}{p+\mu} \frac{a \mathfrak{v}}{1+\mathfrak{v}}-y\right) \mathfrak{F}^{\prime \prime}(y) d y \mid \\
& \quad \leq\left(\frac{1+\lambda}{p+\mu}-\frac{\mu \mathfrak{v}}{p+\mu}+\frac{1}{p+\mu} \frac{a \mathfrak{v}}{1+\mathfrak{v}}\right)^{2}\left\|\mathfrak{F}^{\prime \prime}\right\|,
\end{aligned}
$$

we have

$$
\begin{aligned}
\left|\tilde{\mathfrak{\Omega}}_{p, a}^{(\lambda, \mu)}(\mathfrak{F} ; \mathfrak{v})-\mathfrak{F}(\mathfrak{v})\right| \leq & \mathfrak{Q}_{p, a}^{(\lambda, \mu)}\left((t-\mathfrak{v})^{2} ; \mathfrak{v}\right)+\left(\frac{1+\lambda}{p+\mu}-\frac{\mu \mathfrak{v}}{p+\mu}\right. \\
& \left.+\frac{1}{p+\mu} \frac{a \mathfrak{v}}{1+\mathfrak{b}}\right)^{2}\left\|\mathfrak{F}^{\prime \prime}\right\| .
\end{aligned}
$$

Now, by Corollary 4 (b), we get

$$
\left|\tilde{\mathfrak{Q}}_{p, a}^{(\lambda, \mu)}(\mathfrak{F} ; \mathfrak{b})-\mathfrak{E}(\mathfrak{b})\right| \leq \phi_{p, a}^{(\lambda, \mu)}(\mathfrak{b})\left\|\mathfrak{E}^{\prime \prime}\right\| .
$$

By (27), we get

$$
\begin{aligned}
& \left|\mathfrak{Q}_{p, a}^{(\lambda, \mu)}(\mathfrak{h} ; \mathfrak{b})-\mathfrak{h}(\mathfrak{b})\right| \\
& \leq\left|\tilde{\mathbf{\Omega}}_{p, a}^{(\lambda, \mu)}(\mathfrak{h}-\mathfrak{F} ; \mathfrak{v})\right|+|(\mathfrak{h}-\mathfrak{E})(\mathfrak{b})| \\
& +\left|\tilde{\mathfrak{\Omega}}_{p, a}^{(\lambda, \mu)}(\mathfrak{F} ; \mathfrak{b})-\mathfrak{E}(\mathfrak{b})\right| \\
& +\left|\mathfrak{h}\left(\frac{1+\lambda}{p+\mu}+\frac{p \mathfrak{v}}{p+\mu}+\frac{1}{p+\mu} \frac{a \mathfrak{v}}{1+\mathfrak{v}}\right)-\mathfrak{h}(\mathfrak{v})\right| .
\end{aligned}
$$

Since $\left|\tilde{\mathfrak{\Omega}}_{p, a}^{(\lambda, \mu)}(\mathfrak{h} ; \mathfrak{b})\right| \leq 3\|\mathfrak{h}\|$, we get

$$
\begin{aligned}
& \left|\mathfrak{Q}_{p, a}^{(\lambda, \mu)}(\mathfrak{h} ; \mathfrak{v})-\mathfrak{h}(\mathfrak{b})\right| \\
& \quad \leq 4\|\mathfrak{h}-\mathfrak{E}\|+\left|\tilde{\mathfrak{Q}}_{p, a}^{(\lambda, \mu)}(\mathfrak{F} ; \mathfrak{b})-\mathfrak{E}(\mathfrak{b})\right| \\
& \quad+\left|\mathfrak{h}\left(\frac{1+\lambda}{p+\mu}+\frac{p \mathfrak{v}}{p+\mu}+\frac{1}{p+\mu} \frac{a \mathfrak{v}}{1+\mathfrak{b}}\right)-\mathfrak{h}(\mathfrak{b})\right|
\end{aligned}
$$


From (33), we get

$$
\begin{aligned}
\left|\mathfrak{Q}_{p, a}^{(\lambda, \mu)}(\mathfrak{h} ; \mathfrak{b})-\mathfrak{h}(\mathfrak{b})\right| \leq & 4\|\mathfrak{h}-\mathfrak{E}\|+\phi_{p, a}^{(\lambda, \mu)}(\mathfrak{v})\left\|\mathfrak{F}^{\prime \prime}\right\|+\omega_{1}\left(\mathfrak{h}: \frac{1+\lambda}{p+\mu}\right. \\
& \left.-\frac{\mu \mathfrak{v}}{p+\mu}+\frac{1}{p+\mu} \frac{a \mathfrak{v}}{1+\mathfrak{b}}\right) .
\end{aligned}
$$

Now, taking the infimum over all $\mathfrak{E} \in C_{B}^{2}[0, \infty)$, we obtain

$$
\begin{aligned}
\left|\mathfrak{Q}_{p, a}^{(\lambda, \mu)}(\mathfrak{h} ; \mathfrak{b})-\mathfrak{h}(\mathfrak{b})\right| \leq & 4 K_{2}\left(\mathfrak{h} ; \phi_{p, a}^{(\lambda, \mu)}(\mathfrak{v})\right)+\omega_{1}\left(f: \frac{1+\lambda}{p+\mu}\right. \\
& \left.-\frac{\mu \mathfrak{v}}{p+\mu}+\frac{1}{p+\mu} \frac{a \mathfrak{v}}{1+\mathfrak{b}}\right) .
\end{aligned}
$$

Hence, by using (22), we get the result.

For our next result, we consider the functions belonging to the Lipschitz class:

$$
\operatorname{lip}_{\mathscr{M}}(\gamma)=\left\{\mathfrak{h} \in C_{B}[0, \infty):|\mathfrak{h}(t)-\mathfrak{h}(\mathfrak{v})| \leq \mathscr{M} \frac{|t-\mathfrak{v}|^{\gamma}}{(t+\mathfrak{v})^{\gamma / 2}}\right\}
$$

where $\mathscr{M}>0$ and $0<\gamma \leq 1 ; \mathfrak{v}, t \in 0, \infty)$.

Theorem 7. For $\mathfrak{h} \in \operatorname{lip}_{\mathscr{M}}(\gamma)$, we have

$$
\left|\mathfrak{Q}_{p, a}^{(\lambda, \mu)}(\mathfrak{h} ; \mathfrak{v})-\mathfrak{h}(\mathfrak{v})\right| \leq \mathscr{M}\left(\frac{\varphi_{p}^{(\lambda, \mu)}(\mathfrak{b})}{\mathfrak{v}}\right)^{\gamma / 2}
$$

where $\varphi_{p, a}^{(\lambda, \mu)}(\mathfrak{v})=\mathbf{\Omega}_{p, a}^{(\lambda, \mu)}\left(\left(e_{1}-\mathfrak{v}\right)^{2} ; \mathfrak{v}\right)$

Proof. First, we prove for $\gamma=1$. For $\mathfrak{h} \in \operatorname{lip}_{\mathscr{M}}(\gamma)$, we get

$$
\begin{aligned}
& \left|\mathfrak{Q}_{p, a}^{(\lambda, \mu)}(\mathfrak{h} ; \mathfrak{b})-\mathfrak{h}(\mathfrak{v})\right| \\
& \quad \leq p \sum_{k=0}^{\infty} W_{p, k}^{a}(\mathfrak{v}) \int_{0}^{\infty} s_{p, k}(t)\left|\mathfrak{h}\left(\frac{p t+\lambda}{p+\mu}\right)-\mathfrak{h}(\mathfrak{b})\right| d t \\
& \quad \leq M p \sum_{k=0}^{\infty} W_{p, k}^{a}(\mathfrak{v}) \int_{0}^{\infty} s_{p, k}(t) \frac{|((p t+\lambda) /(p+\mu))-\mathfrak{v}|}{\sqrt{((p t+\lambda) /(p+\mu))+\mathfrak{v}}} d t .
\end{aligned}
$$

Since $\sqrt{\mathfrak{b}}<\sqrt{((p t+\lambda) /(p+\mu))+\mathfrak{b}}$, we get by the Cauchy-Schwarz inequality:

$$
\begin{aligned}
& \left|\boldsymbol{Q}_{p, a}^{(\lambda, \mu)}(\mathfrak{h} ; \mathfrak{b})-\mathfrak{h}(\mathfrak{b})\right| \\
& \quad \leq \frac{\mathscr{M}}{\sqrt{\mathfrak{b}}} p \sum_{k=0}^{\infty} W_{p, k}^{a}(\mathfrak{b}) \int_{0}^{\infty} s_{n, k}(t)\left|\frac{p t+\lambda}{p+\mu}-u\right| d t \\
& \quad=\frac{\mathscr{M}}{\sqrt{\mathfrak{b}}} \mathbb{Q}_{p, a}^{(\lambda, \mu)}\left(\left(e_{1}-\mathfrak{b}\right)^{2} ; \mathfrak{b}\right) \leq \mathscr{M} \sqrt{\frac{\varphi_{p, a}^{(\lambda, \mu)}(\mathfrak{v})}{\mathfrak{b}}} .
\end{aligned}
$$

For $0<\gamma<1$, applying Hölder's inequality, we get

$$
\begin{aligned}
& \left|\mathfrak{Q}_{p, a}^{(\lambda, \mu)}(\mathfrak{h} ; \mathfrak{b})-\mathfrak{h}(\mathfrak{b})\right| \\
& \quad \leq p \sum_{k=0}^{\infty} W_{p, k}^{a}(\mathfrak{b}) \int_{0}^{\infty} s_{p, k}(t)\left|\mathfrak{h}\left(\frac{p t+\lambda}{p+\mu}\right)-\mathfrak{h}(\mathfrak{b})\right| d t \\
& \quad \leq p \sum_{k=0}^{\infty}\left\{W_{p, k}^{a}(\mathfrak{v})\left(\int_{0}^{\infty} s_{p, k}(t)\left|\mathfrak{h}\left(\frac{p t+\lambda}{p+\mu}\right)-\mathfrak{h}(\mathfrak{b})\right| d t\right)^{1 / \gamma}\right\}^{\gamma} \\
& \quad \leq p \sum_{k=0}^{\infty}\left\{W_{p, k}^{a}(\mathfrak{v}) \int_{0}^{\infty} s_{p, k}(t)\left|\mathfrak{h}\left(\frac{p t+\lambda}{p+\mu}\right)-\mathfrak{h}(\mathfrak{v})\right|^{1 / \gamma} d t\right\}^{\gamma} .
\end{aligned}
$$

Since $\mathfrak{h} \in \operatorname{lip}_{M}(\gamma)$, we have

$$
\begin{aligned}
& \mid \mathbf{Q}_{p, a}^{(\lambda, \mu)}(\mathfrak{h} ; \mathfrak{v})-\mathfrak{h}(\mathfrak{v}) \\
& \quad \leq \frac{\mathscr{M}}{\mathfrak{b}^{\gamma / 2}}\left\{p \sum_{k=0}^{\infty} W_{p, k}^{a}(\mathfrak{v}) \int_{0}^{\infty} s_{p, k}(t)\left|\frac{p t+\lambda}{p+\mu}-\mathfrak{v}\right| d t\right\}^{\gamma} \\
& \quad=\frac{\mathscr{M}}{\mathfrak{b}^{\gamma / 2}} \mathbf{Q}_{p, a}^{(\lambda, \mu)}\left(\left|e_{1}-\mathfrak{v}\right| ; \mathfrak{v}\right)^{\gamma}=\frac{\mathscr{M}}{\mathfrak{b}^{\gamma / 2}} \mathbf{Q}_{p, a}^{(\lambda, \mu)}\left(\left(e_{1}-\mathfrak{v}\right)^{2} ; \mathfrak{v}\right)^{\gamma} \\
& \quad \leq \mathscr{M}\left(\sqrt{\frac{\varphi_{p, a}^{(\lambda, \mu)}(\mathfrak{b})}{\mathfrak{v}}}\right)^{\gamma} .
\end{aligned}
$$

Therefore, we get (39).

Next, we obtain a Voronovskaja-type asymptotic formula.

Theorem 8. If $\mathfrak{h}^{\prime \prime}$ exists at a point $\left.\mathfrak{v} \in 0, \infty\right)$ for $\mathfrak{h} \in C_{\gamma}[0, \infty)$, then

$$
\begin{aligned}
& \lim _{p \longrightarrow \infty} p\left(\mathfrak{Q}_{p, a}^{(\lambda, \mu)}(\mathfrak{h} \simeq \mathfrak{v})-\mathfrak{h}(\mathfrak{v})\right) \\
& \quad=\left(1+\lambda-\mu \mathfrak{v}+\frac{a \mathfrak{v}}{1+\mathfrak{b}}\right) \mathfrak{h}^{\prime}(\mathfrak{v})+\frac{\mathfrak{v}}{2}(2+\mathfrak{v}) \mathfrak{h}^{\prime \prime}(\mathfrak{v}) .
\end{aligned}
$$

Proof. From Taylor's expansion of $\mathfrak{v}$, we may write

$\mathfrak{h}(t)=\mathfrak{h}(\mathfrak{b})+(t-\mathfrak{v}) \mathfrak{h}^{\prime}(\mathfrak{v})+\frac{1}{2}(t-\mathfrak{v})^{2} \mathfrak{h}^{\prime \prime}(\mathfrak{v})+R(t, \mathfrak{v})(t-\mathfrak{v})^{2}$ 
where $R(t, \mathfrak{v}) \longrightarrow 0(t \longrightarrow \mathfrak{v})$. By operating $\mathbf{\Omega}_{p, a}^{(\lambda, \mu)}$, we obtain

$$
\begin{aligned}
\mathfrak{\Omega}_{p, a}^{(\lambda, \mu)}(\mathfrak{h} \simeq \mathfrak{b})-\mathfrak{h}(\mathfrak{b})= & \mathfrak{\Omega}_{p, a}^{(\lambda, \mu)}((t-\mathfrak{v}) ; \mathfrak{v}) \mathfrak{h}^{\prime}(\mathfrak{v}) \\
& +\mathfrak{Q}_{p, a}^{(\lambda, \mu)}\left((t-\mathfrak{v})^{2} ; \mathfrak{b}\right) \frac{\mathfrak{h}^{\prime \prime}(\mathfrak{v})}{2} \\
& +\mathfrak{\Omega}_{p, a}^{(\lambda, \mu)}\left(R(t, \mathfrak{v})(t-\mathfrak{b})^{2} ; \mathfrak{b}\right) .
\end{aligned}
$$

By the Cauchy-Schwarz inequality, we get

$$
\begin{aligned}
& \boldsymbol{Q}_{p, a}^{(\lambda, \mu)}\left(R(t, \mathfrak{v})(t-\mathfrak{v})^{2} ; \mathfrak{b}\right) \\
& \quad \leq\left(\boldsymbol{Q}_{p, a}^{(\lambda, \mu)}\left(R^{2}(t, \mathfrak{v}) ; \mathfrak{b}\right)\right)^{1 / 2}\left(\boldsymbol{\Omega}_{p, a}^{(\lambda, \mu)}\left((t-\mathfrak{v})^{4} ; \mathfrak{v}\right)\right)^{1 / 2} .
\end{aligned}
$$

Since $\mathfrak{\Omega}_{p, a}^{(\lambda, \mu)}(\mathfrak{h} \simeq \mathfrak{v}) \longrightarrow \mathfrak{h}(\mathfrak{v})$, we get

$$
\begin{gathered}
\lim _{p \longrightarrow \infty} \mathfrak{Q}_{p, a}^{(\lambda, \mu)}\left(R^{2}(t, \mathfrak{v}) ; \mathfrak{b}\right)=R^{2}(\mathfrak{v}, \mathfrak{v})=0, \\
\lim _{p \longrightarrow \infty} p \mathfrak{Q}_{p, a}^{(\lambda, \mu)}\left(R(t, \mathfrak{v})(t-\mathfrak{v})^{2} ; \mathfrak{v}\right)=0 .
\end{gathered}
$$

Now, combining the above equations and using Corollary 5 , we get

$$
\begin{aligned}
& \lim _{p \longrightarrow \infty} p\left(\boldsymbol{\Omega}_{p, a}^{(\lambda, \mu)}(\mathfrak{h} \simeq \mathfrak{b})-\mathfrak{h}(\mathfrak{b})\right) \\
& =\lim _{p \longrightarrow \infty} p\left(\mathfrak{\Omega}_{p, a}^{(\lambda, \mu)}((t-\mathfrak{v}) ; \mathfrak{v})\right) \mathfrak{h}^{\prime}(\mathfrak{v}) \\
& +\lim _{p \longrightarrow \infty} p\left(\boldsymbol{\Omega}_{p, a}^{(\lambda, \mu)}\left((t-\mathfrak{v})^{2} ; \mathfrak{v}\right)\right) \frac{\mathfrak{h}^{\prime \prime}(\mathfrak{v})}{2} \\
& +\lim _{p \longrightarrow \infty} p\left(\mathfrak{\Omega}_{p, a}^{(\lambda, \mu)}\left(R(t, \mathfrak{v})(t-\mathfrak{v})^{2} ; \mathfrak{b}\right)\right) \\
& =\left(1+\lambda-\mu \mathfrak{v}+\frac{a \mathfrak{v}}{1+\mathfrak{b}}\right) \mathfrak{h}^{\prime}(\mathfrak{v})+\frac{\mathfrak{v}}{2}(2+\mathfrak{v}) \mathfrak{h}^{\prime \prime}(\mathfrak{v}) \text {. }
\end{aligned}
$$

Let $B_{\sigma}[0, \infty)=\left\{\mathfrak{h}:[0, \infty) \longrightarrow \mathbb{R}|| \mathfrak{h}(\mathfrak{v}) \mid \leq \mathscr{K}_{\mathfrak{h}} \sigma(\mathfrak{b}), \mathfrak{v} \geq 0\right\}$ , where $\mathscr{K}_{\mathfrak{h}}$ is a constant which depends only on $\mathfrak{h}$, and

$$
\|\mathfrak{h}\|_{\sigma}=\sup _{\mathfrak{b} \in 0, \infty)} \frac{|\mathfrak{h}(\mathfrak{b})|}{\sigma(\mathfrak{b})} .
$$

Also, let $C_{\sigma}[0, \infty)=\left\{\mathfrak{h} \in B_{\sigma}[0, \infty): \mathfrak{h}\right.$ be continuous on [ $0, \infty)\}$, and

$$
C_{\sigma}^{0}[0, \infty)=\left\{\mathfrak{h} \in C_{\sigma}[0, \infty): \lim _{\mathfrak{v} \longrightarrow \infty} \frac{|\mathfrak{h}(\mathfrak{b})|}{\sigma(\mathfrak{b})} \text { exists }\right\},
$$

where $\sigma(\mathfrak{b})=1+\mathfrak{v}^{2}$.

The weighted modulus of continuity [14] is defined by

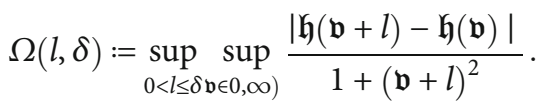

Lemma 9 (see [14]). Let $\mathfrak{h} \in C_{\sigma}^{0}[0, \infty)$. Then, (i) $\Omega(l, \delta)$ is a monotone increasing function of $\delta$

(ii) $\Omega(l, \delta) \longrightarrow 0$ as $\delta \longrightarrow 0$

(iii) $\Omega(l, k \delta) \leq k \Omega(l, \delta)$ for each $k \in \mathbb{N}$

(iv) $\Omega(l, \alpha \delta) \leq(1+\alpha) \Omega(l, \delta)$ for each $\alpha \in \mathbb{R}^{+}$

Theorem 10. For $\mathfrak{h} \in C_{\sigma}^{0}[0, \infty)$, we have

$$
\sup _{\mathfrak{b} \in 0, \infty)} \frac{\left|\boldsymbol{Q}_{p, a}^{(\lambda, \mu)}(\mathfrak{h} ; \mathfrak{v})-\mathfrak{h}(\mathfrak{b})\right|}{\left(1+\mathfrak{b}^{2}\right)^{5 / 2}} \leq M \Omega\left(l, \frac{1}{p}\right), M>0
$$

Proof. By Lemma 9, we have

$$
\begin{aligned}
|\mathfrak{h}(t)-\mathfrak{h}(\mathfrak{b})| & \leq\left(1+(\mathfrak{b}+|t-\mathfrak{v}|)^{2} \Omega(l,|t-\mathfrak{v}|)\right. \\
& \leq 2\left(1+\mathfrak{v}^{2}\right)\left(1+(t-\mathfrak{v})^{2}\right)\left(1+\frac{|t-\mathfrak{v}|}{\delta}\right) \Omega(l, \delta) .
\end{aligned}
$$

Operating $\mathbf{Q}_{p, a}^{(\lambda, \mu)}$, we get

$$
\begin{aligned}
& \left|\mathfrak{Q}_{p, a}^{(\lambda, \mu)}(\mathfrak{h} ; \mathfrak{v})-\mathfrak{h}(\mathfrak{b})\right| \\
& \leq 2\left(1+\mathfrak{v}^{2}\right) \Omega(l, \delta)\left\{1+\mathfrak{Q}_{p, a}^{(\lambda, \mu)}\left((t-\mathfrak{b})^{2} ; \mathfrak{b}\right)\right. \\
& \left.\quad+\mathfrak{Q}_{p, a}^{(\lambda, \mu)}\left(1+(t-\mathfrak{v})^{2} \frac{|\mathfrak{v}-t|}{\delta} ; \mathfrak{b}\right)\right\}
\end{aligned}
$$

Using a second-order central moment, we get

$\mathbf{Q}_{p, a}^{(\lambda, \mu)}\left((t-\mathfrak{v})^{2} ; \mathfrak{v}\right) \leq M_{1} \frac{\left(1+\mathfrak{v}^{2}\right)}{(p+\mu)} \leq M_{1} \frac{\left(1+\mathfrak{v}^{2}\right)}{p}, M_{1}>0$.

Applying the Cauchy-Schwarz inequality, we obtain

$$
\begin{aligned}
& \mathfrak{Q}_{p, a}^{(\lambda, \mu)}\left(1+(t-\mathfrak{v})^{2} \frac{|\mathfrak{v}-t|}{\delta} ; u\right) \\
& \leq \frac{1}{\delta}\left(\mathbf{Q}_{p, a}^{(\lambda, \mu)}\left((t-\mathfrak{v})^{2} ; \mathfrak{b}\right)\right)^{1 / 2} \\
& \quad+\frac{1}{\delta}\left(\mathbf{Q}_{p, a}^{(\lambda, \mu)}\left((t-\mathfrak{v})^{4} ; \mathfrak{v}\right)\right)^{1 / 2}\left(\mathfrak{Q}_{p, a}^{(\lambda, \mu)}\left((t-\mathfrak{v})^{2} ; \mathfrak{v}\right)\right)^{1 / 2}
\end{aligned}
$$

Again, using the central moment of order 4, we get

$$
\left(\boldsymbol{R}_{p, a}^{(\lambda, \mu)}\left((t-\mathfrak{v})^{4} ; \mathfrak{v}\right)\right)^{1 / 2} \leq M_{2} \frac{\left(1+\mathfrak{v}^{2}\right)}{(p+\mu)} \leq M_{2} \frac{\left(1+\mathfrak{v}^{2}\right)}{p}, M_{2}>0
$$

Combining the estimates (55)-(58) and choosing $M=2$ $\left(1+M_{1}+\sqrt{M_{1}}+M_{2} \sqrt{M_{1}}\right), \delta=1 / \sqrt{p}$, we get the required result. 


\section{4. $q$-Statistical Convergence}

Defining a $q$-analog of the Cesàro matrix $C_{1}$ is not unique (see $[15,16])$. Here, we consider the $q$-Cesàro matrix, $C_{1}$ $(q)=\left(c_{n k}^{1}\left(q^{k}\right)\right)_{n, k=0}^{\infty}$, defined by

$$
c_{n k}^{1}\left(q^{k}\right)=\left(\begin{array}{ll}
\frac{q^{k}}{[n+1]_{q}}, & \text { if } k \leq n, \\
0, & \text { otherwise, }
\end{array}\right.
$$

which is regular for $q \geq 1$.

Let $\mathscr{K} \subseteq \mathbb{N}$ (the set of natural numbers). Then, $\delta(\mathscr{K})=$ $\lim _{r}(1 / r) \#\{k \leq r: k \in \mathscr{K}\}$ is called the asymptotic density of $\mathscr{K}$, where \# denotes the cardinality of the enclosed set. A sequence $\eta=\left(\eta_{k}\right)$ is called statistically convergent to the number $\mathfrak{g}$ if $\delta\left(\mathscr{K}_{\varepsilon}\right)=0$ for each $\varepsilon>0$, where $\mathscr{K}_{\varepsilon}=\{k \leq r$ $\left.:\left|\eta_{k}-\mathfrak{g}\right|>\varepsilon\right\}$ (see [17]).

Recently, Aktuğlu and Bekar [16] defined q-density and $q$-statistical convergence. The $q$-density is defined by

$$
\begin{aligned}
\delta_{q}(\mathscr{K}) & =\delta_{C_{1}^{q}}(\mathscr{K})=\lim _{n \longrightarrow \infty} \inf _{n \rightarrow \infty}\left(C_{1}^{q} \chi_{\mathscr{K}}\right)_{n} \\
& =\lim _{n \longrightarrow \infty} \sum_{k \in K} \frac{q^{k-1}}{[n]}, q \geq 1 .
\end{aligned}
$$

A sequence $\eta=\left(\eta_{k}\right)$ is said to be $q$-statistically convergent to the number $\mathscr{L}$ if $\delta_{q}\left(\mathscr{K}_{\varepsilon}\right)=0$, where $\mathscr{K}_{\varepsilon}=\{k \leq n: \mid$ $\left.\eta_{k}-\mathscr{L} \mid \geq \varepsilon\right\}$ for every $\varepsilon>0$. That is, for each $\varepsilon>0$,

$$
\lim _{n} \frac{1}{[n]} \#\left\{k \leq n: q^{k-1}\left|\eta_{k}-\mathscr{L}\right| \geq \varepsilon\right\}=0,
$$

and we write $S t_{q}-\lim \eta_{k}=\mathscr{L}$.

If $\delta(\mathscr{K})=0$ for an infinite set $\mathscr{K}$, then $\delta_{q}(\mathscr{K})=0$; hence, statistical convergence implies $q$-statistical convergence but not conversely (c.f. [16, Example 15]). Recently in [18], authors proved Korovkin's type theorem via q-statistical convergence. Using the same technique we prove the following theorem.

Theorem 11. For all $\mathfrak{h} \in C_{\rho}^{0}$, we have

$$
S t_{q}-\lim _{p}\left\|\mathfrak{Q}_{p, a}^{(\lambda, \mu)}(\mathfrak{h} ; \mathfrak{b})-\mathfrak{h}(\mathfrak{b})\right\|_{\sigma}=0, \mathfrak{v} \in[0, \infty)
$$

Proof. It is sufficient to show that $S t_{q}-\lim _{p} \| \boldsymbol{\Omega}_{p, a}^{(\lambda, \mu)}\left(e_{i} ; \mathfrak{b}\right)-$ $e_{i} \|_{\sigma}=0$, for $i=0,1,2$, where $e_{i}(\mathfrak{v})=\mathfrak{v}^{i}$. It is clear that

$$
S t_{q}-\lim _{p}\left\|\mathfrak{Q}_{p, a}^{(\lambda, \mu)}\left(e_{0} ; \mathfrak{b}\right)-e_{0}\right\|_{\sigma}=0 .
$$

$$
\begin{aligned}
\left\|\mathfrak{Q}_{p, a}^{(\lambda, \mu)}(t ; \mathfrak{v})-\mathfrak{v}\right\|_{\sigma}= & \sup _{u \in 0, \infty)} \frac{1}{1+\mathfrak{v}^{2}} \mid \frac{1}{p+\mu}\left(p \mathfrak{v}+\frac{a \mathfrak{v}}{1+\mathfrak{v}}\right. \\
+1+\lambda)-\mathfrak{v} & \leq \frac{1}{p+\mu}|-\mu+1+\lambda+a| .
\end{aligned}
$$

For $\varepsilon>0$, define the sets:

$$
\begin{gathered}
\mathscr{E}_{1}:=\left\{p \in \mathbb{N}:\left\|\boldsymbol{\Omega}_{p, a}^{(\lambda, \mu)}\left(e_{1} ; \mathfrak{b}\right)-e_{1}\right\|_{\sigma} \geq \varepsilon\right\}, \\
\mathscr{E}_{2}:=\left\{p \in \mathbb{N}:\left|\frac{1+\lambda-\mu+a}{(p+\mu)}\right| \geq \varepsilon\right\} .
\end{gathered}
$$

Then,

$\delta_{q}\left(\mathscr{E}_{2}\right)=\lim _{p \longrightarrow \infty} \inf \left(C_{1}^{q} \chi_{\mathscr{E}_{2}}\right)_{p}=\lim _{p \longrightarrow \infty} \inf \sum_{k \in \mathscr{E}_{2}} \frac{q^{k-1}}{[p]}=0$

Since $\mathscr{E}_{1} \subseteq \mathscr{E}_{2}$, we have $\delta_{q}\left(\mathscr{E}_{1}\right) \leq \delta_{q}\left(\mathscr{E}_{2}\right)$. Hence,

$$
S t_{q}-\lim _{p}\left\|\boldsymbol{\mathfrak { R }}_{p, a}^{(\lambda, \mu)}\left(e_{1} ; \mathfrak{b}\right)-e_{1}\right\|_{\sigma}=0
$$

Again, by Corollary 2 (iii), we obtain

$$
\begin{aligned}
& \| \mathfrak{Q}_{p, a}^{(\lambda, \mu)}\left(t^{2} ; \mathfrak{v}\right)-\mathfrak{v}^{2}||_{\sigma} \\
& \leq \sup _{\mathfrak{b} \in 0, \infty)} \frac{1}{1+\mathfrak{v}^{2}}\left|\frac{1}{(p+\mu)^{2}}\left\{p+\mu^{2}+\frac{a^{2}}{(1+\mathfrak{v})^{2}}-\frac{2 a \mu}{(1+\mathfrak{v})}\right\} \mathfrak{v}^{2}\right| \\
& \quad+\sup _{\mathfrak{v} \in 0, \infty)} \frac{1}{1+\mathfrak{v}^{2}} \mid \frac{2}{(p+\mu)^{2}}\left(p-\mu-\lambda \mu+\frac{(2+\lambda) a}{(1+\mathfrak{v})}\right) \mathfrak{v} \\
& \quad+\frac{1}{(p+\mu)^{2}}\left(\lambda^{2}+2 \lambda+2\right) \mid \leq \frac{1}{(p+\mu)^{2}}\left\{p+\mu^{2}+a^{2}-2 a \mu\right\} \\
& \quad+\frac{2}{(p+\mu)^{2}}(p-\mu-\lambda \mu+(2+\lambda) a)+\frac{1}{(p+\mu)^{2}}\left(\lambda^{2}+2 \lambda+2\right) .
\end{aligned}
$$

For $\varepsilon>0$, define the sets:

$$
\begin{aligned}
& \mathfrak{D}_{1}:=\left\{p \in \mathbb{N}:\left\|\mathbb{Q}_{p, a}^{(\lambda, \mu)}\left(e_{2} ; \mathfrak{v}\right)-e_{2}\right\|_{\sigma} \geq \varepsilon\right\}, \\
& \mathfrak{D}_{2}:=\left\{p \in \mathbb{N}:\left(\frac{1}{(p+\mu)^{2}}\left\{p+\mu^{2}+a^{2}-2 a \mu\right\}\right) \geq \frac{\varepsilon}{3}\right\}, \\
& \mathfrak{D}_{3}:=\left\{p \in \mathbb{N}: \frac{2}{(p+\mu)^{2}}(p-\mu-\lambda \mu+(2+\lambda) a) \geq \frac{\varepsilon}{3}\right\}, \\
& \mathfrak{D}_{4}:=\left\{p \in \mathbb{N}: \frac{1}{(p+\mu)^{2}}\left(\lambda^{2}+2 \lambda+2\right) \geq \frac{\varepsilon}{3}\right\} .
\end{aligned}
$$

By Corollary 2 (ii), we have 


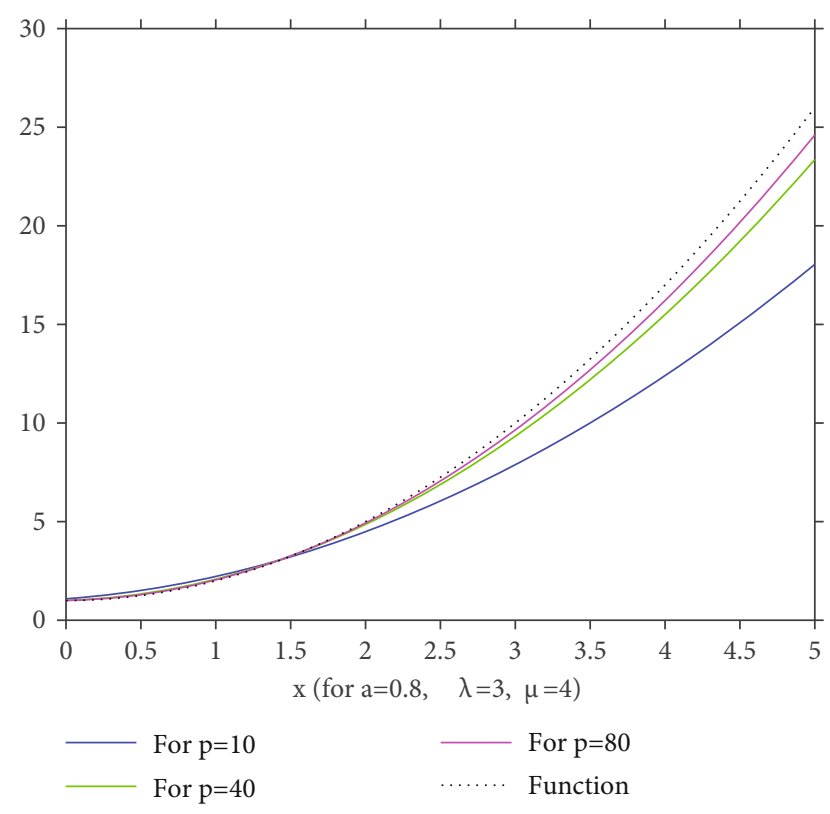

Figure 1: Convergence of the operator towards the function $f(x)$ $=x^{2}+1$.

Then, $\delta_{q}\left(\mathfrak{D}_{2}\right)=0=\delta_{q}\left(\mathfrak{D}_{3}\right)=\delta_{q}\left(\mathfrak{D}_{4}\right)$. Since $\mathfrak{D}_{1} \subseteq \mathfrak{D}_{2} \cup$ $\mathfrak{D}_{3} \cup \mathfrak{D}_{4}$ which implies that $\delta_{q}\left(\mathfrak{D}_{1}\right) \leq \delta_{q}\left(\mathfrak{D}_{2}\right)+\delta_{q}\left(\mathfrak{D}_{3}\right)+$ $\delta_{q}\left(\mathfrak{D}_{4}\right)$,

$$
S t_{q}-\lim _{p}\left\|\mathbf{\Omega}_{p, a}^{(\lambda, \mu)}\left(e_{2} ; \mathfrak{v}\right)-e_{2}\right\|_{\rho}=0
$$

Hence, the proof is completed.

Example 12. Let $\eta=\left(\eta_{p}\right)$ be defined by

$$
\eta_{p}=\left(\begin{array}{c}
1\left(2^{2 n} \text { times }\right) \\
0\left(2^{2 n-1} \text { times }\right)
\end{array} n=0,1,2, \cdots\right.
$$

That is, 1 occurs $2^{2 n}$ times and 0 occurs $2^{2 n-1}$ times $(n$ $=0,1,2, \cdots)$, respectively. Let $\mathscr{K}=\left\{k \in \mathbb{N}: \eta_{k}=1\right\}$. Then, $\lim _{n \longrightarrow \infty}\left(C_{1}^{q} \chi_{\mathscr{K}}\right)_{2^{2 n}-1}=0$, i.e., $S t_{q}-\lim \eta_{k}=0$, but $\delta(\mathscr{K})$ does not exist, so $\eta$ is not statistically convergent.

Define $\mathscr{A}_{p}^{(\lambda, \mu)}=\left(1+\eta_{p}\right) \boldsymbol{Q}_{p, a}^{(\lambda, \mu)}$, where it is defined by (71). Then, obviously $s t-\lim _{p}\left\|\mathscr{A}_{p}^{(\lambda, \mu)}\left(e_{i} ; \mathfrak{b}\right)-e_{i}\right\|_{\sigma}=0(i=0,1,2)$. Applying the above theorem, we have

$$
S t_{q}-\lim _{p}\left\|\mathscr{A}_{p}^{(\lambda, \mu)}(\mathfrak{h} ; \mathfrak{b})-\mathfrak{h}\right\|_{\sigma}=0 \text { for all } \mathfrak{h} \in C_{\rho}^{0}
$$

On the other hand, since $\eta=\left(\eta_{p}\right)$ is $q$-statistically convergent but neither convergent nor statistically convergent, the sequence $\left(\mathscr{A}_{p}^{(\lambda, \mu)}\right)$ can not be convergent, while it is $q$ -statistically convergent.

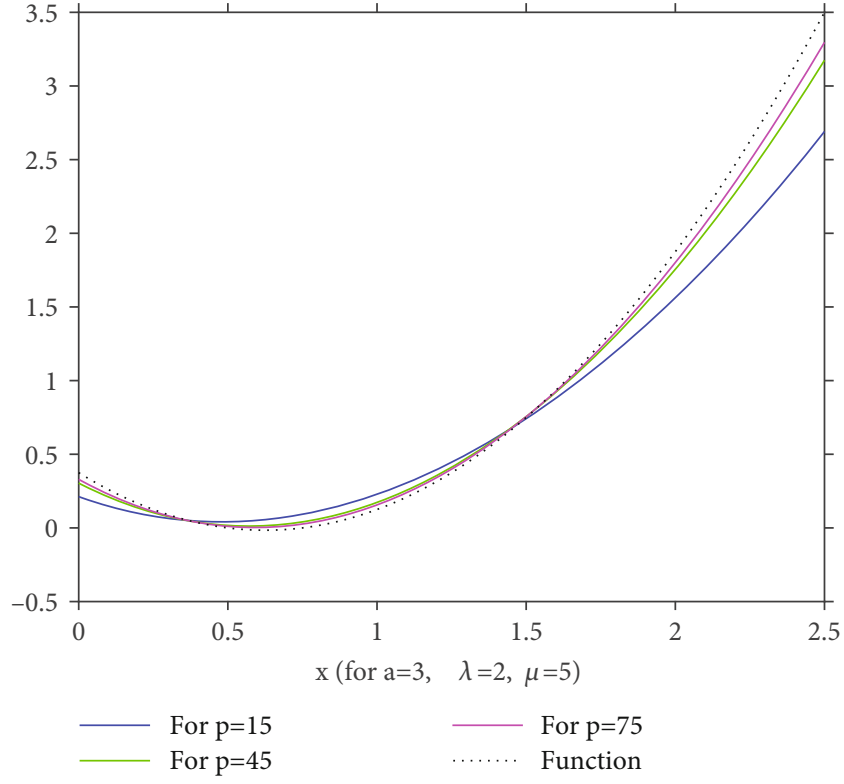

FIGURe 2: Convergence of the operator towards the function $f(x)$ $=(x-(1 / 2))(x-(3 / 4))$.

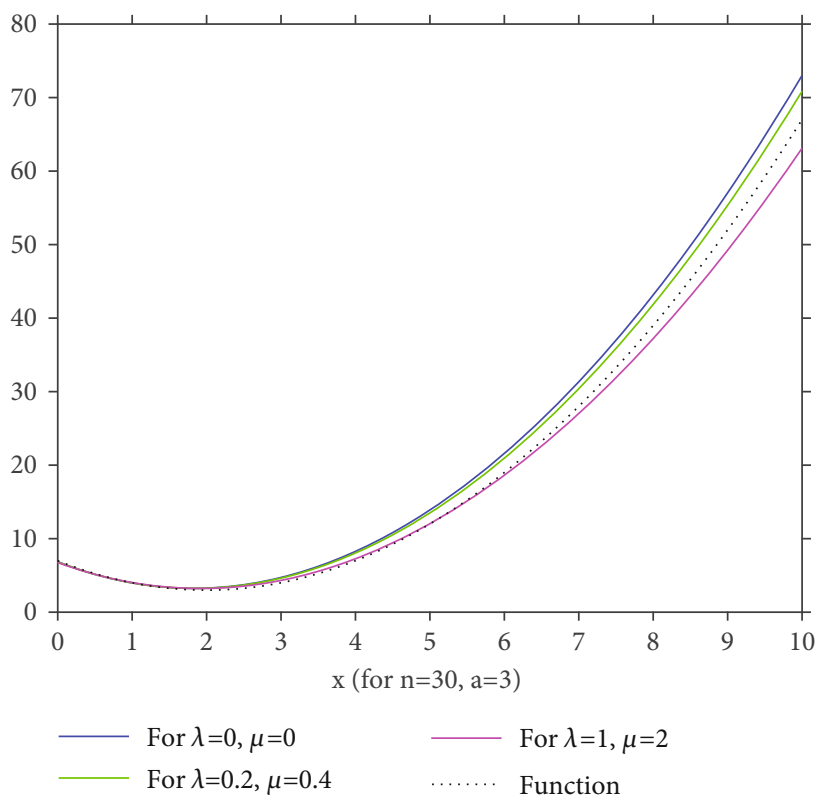

FIGURE 3: Comparison of convergence of the operator.

\section{Graphical Analysis}

In this section, we will give some numerical examples with illustrative graphics with the help of MATLAB.

Example 13. Let $f(x)=x^{2}+1, \lambda=3, \mu=4, a=0.8$, and $p \in$ $\{10,40,80\}$. The convergence of the operator towards the function $f(x)$ is shown in Figure 1 .

Example 14. Let $f(x)=(x-(1 / 2))(x-(3 / 4)), \lambda=2, \mu=5, a$ $=3$, and $p \in\{15,45,75\}$. The convergence of the operator towards the function $f(x)$ is shown in Figure 2. 
From these examples, we observe that the approximation of function by the operators becomes better when we take larger values of $n$.

Notice that for $\lambda=\mu=0$, the operators (5) reduce to operators (4).

Example 15. Let $f(x)=x^{2}-4 x+7$. For $a=3, p=30$, comparison of convergence of the constructed operator (5) (green and pink) with the previously defined operator (4) (blue) is shown in Figure 3. From this figure, it is clear that the constructed operator gives a better approximation to $f(x)$ than the previously defined operator.

\section{Data Availability}

No data were used to support this study.

\section{Conflicts of Interest}

The authors declare that they have no conflicts of interest.

\section{Authors' Contributions}

All authors contributed equally to writing this paper. All authors read and approved the manuscript.

\section{Acknowledgments}

This work is supported by the Natural Science Foundation of Fujian Province of China (Grant No. 2020J01783), the Project for High-level Talent Innovation and Entrepreneurship of Quanzhou (Grant No. 2018C087R), and the Program for New Century Excellent Talents in Fujian Province University.

\section{References}

[1] S. N. Bernstein, "Démonstation du théorème de Weierstrass fondée sur le calcul de probabilités," Communication Society Mathematical Kharkov, vol. 13, 1913 pages, 1912.

[2] O. Szász, "Generalization of S. Bernstein's polynomials to the infinite interval," Journal of Research of the National Bureau of Standards, vol. 45, no. 3, pp. 239-245, 1950.

[3] G. M. Mirakjan, "Approximation of continuous functions with the aid of polynomials (Russian)," Doklady Akademii Nauk SSSR, vol. 31, pp. 201-205, 1941.

[4] L. V. Kantorovich, "Sur certains développements suivant les polynômes de la forme de S," Comptes Rendus de l'Academie des Sciences de l'URSSR, vol. 20, pp. 563-568, 1930.

[5] J. L. Durrmeyer, "Une formule d' inversion de la Transformee de Laplace," in Applications a la Theorie des Moments, These de 3e Cycle, Faculte des Sciences de l' Universite de Paris, 1967.

[6] D. D. Stancu, "Approximation of functions by a new class of linear polynomial operators," Revue Roumaine de Mathématiques Pures et Appliquées, vol. 13, pp. 1173-1194, 1968.

[7] N. L. Braha, T. Mansour, and M. Mursaleen, "Some properties of Kantorovich-Stancu-type generalization of Szász operators including Brenke-type polynomials via power series summability method," Journal of Function Spaces, vol. 2020, Article ID 3480607, 15 pages, 2020.
[8] S. A. Mohiuddine, A. Kajla, M. Mursaleen, and M. A. Alghamdi, "Blending type approximation by $\tau$-BaskakovDurrmeyer type hybrid operators," Advances in Difference Equations, vol. 2020, no. 1, 12 pages, 2020.

[9] S. Rahman, M. Mursaleen, and A. Khan, "A Kantorovich variant of Lupas-Stancu operators based on Polya distribution with error estimation," Revista de la Real Academia de Ciencias Exactas, Físicas y Naturales. Serie A. Matemáticas, vol. 114, no. 2, pp. 1-26, 2020.

[10] V. A. Baskakov, "A sequence of linear positive operators in the space of continuous functions," Proceedings of the USSR Academy of Sciences, vol. 113, pp. 249-251, 1957.

[11] R. A. Devore and G. G. Lorentz, Constructive Approximation, Springer, Berlin, 1993.

[12] P. N. Agrawal, V. Gupta, A. S. Kumar, and A. Kajla, "Generalized Baskakov-Szász type operators," Applied Mathematics and Computation, vol. 236, pp. 311-324, 2014.

[13] A. Kilicman, M. Ayman Mursaleen, and A. A. H. A. Al-Abied, "Stancu type Baskakov-Durrmeyer operators and approximation properties," Mathematics, vol. 8, no. 7, p. 1164, 2020.

[14] I. Yüksel and N. Ispir, "Weighted approximation by a certain family of summation integral-type operators," Computers \& Mathematcs with Applications, vol. 52, no. 10-11, pp. 14631470, 2006.

[15] F. Aydin Akgun and B. E. Rhoades, "Properties of some qHausdorff matrices," Applied Mathematics and Computation, vol. 219, no. 14, pp. 7392-7397, 2013.

[16] H. Aktuğlu and Ş. Bekar, "q-Cesaro matrix and q-statistical convergence," Journal Of Computational And Applied Mathematics, vol. 235, no. 16, pp. 4717-4723, 2011.

[17] H. Fast, "Sur la convergence statistique," Colloquium Mathematicum, vol. 2, pp. 241-244, 1949.

[18] A. A. H. A. Al-Abied, M. Ayman Mursaleen, and M. Mursaleen, "Szász type operators involving Charlier polynomials and approximation properties," Filomat, vol. 35, no. 15, pp. 5149-5159, 2021. 